\title{
Characterization of main constituents in potato (Solanum tuberosum) tissues using LIBS
}

\section{Caracterización de los componentes principales en tejidos de papa (Solanum tuberosum) usando LIBS}

\author{
Mayra Garcimuño ${ }^{1,2 *}$, Graciela Bertuccelli ${ }^{1,2}$ \\ 1. Consejo Nacional de Investigaciones Científicas y Técnicas (CONICET) \\ 2. Universidad Nacional del Centro de la Provincia de Buenos Aires (UNCPBA) \\ (*)E-mail: mayrag@exa.unicen.edu.ar
}

Received / Recibido: 21/07/2015 Accepted / Aceptado: 26/08/2015

DOI: $10.7149 /$ OPA.48.3.215

\begin{abstract}
RESUMEN:
La técnica LIBS (Laser-Induced Breakdown Spectroscopy) puede ser una herramienta complementaria a los métodos analíticos comúnmente empleados en estudios de medio ambiente. En este trabajo se presentan los resultados preliminares obtenidos al aplicar LIBS en el análisis de tejidos de papa. Las muestras fueron preparadas usando diferentes partes de este tubérculo. Los elementos mayoritarios han sido detectados y los tejidos diferenciados según la abundancia de los nutrientes. Aunque no se realizó análisis cuantitativo de los componentes, se estudiaron las condiciones óptimas para obtener mediciones confiables y repetibles.
\end{abstract}

Palabras clave: Espectroscopia, Vegetales, LIBS

\section{ABSTRACT:}

LIBS (Laser-Induced Breakdown Spectroscopy) can be a complementary tool to analytical methods commonly employed in environmental studies. This paper presents preliminary results obtained by applying analysis LIBS on potato tissues. Samples were prepared using different parts of this tuber. The major elements have been detected and tissues have been differentiated according to the abundance of nutrients. Although quantitative analysis of components was not performed, the optimal conditions were studied to obtain reliable and repeatable measurements.

Key words: Spectroscopy, Vegetables, LIBS

\section{REFERENCES AND LINKS / REFERENCIAS Y ENLACES}

[1] A. W. Miziolek, V. Palleschi, I. Schechter, Laser-Induced Breakdown Spectroscopy (LIBS) Fundamentals and Applications, Cambridge University Press, New York (2006).

[2] H. R. Griem, Spectral Line Broadening by Plasmas, Academic Press, New York (1974).

[3] D. W. Hahn, N. Omenetto, "Laser-induced Breakdown Spectroscopy (LIBS), Part I: Review of basic diagnostics and plasma-particle interactions: still-challenging issues within the analytical plasma community", App Spectroscopy 64, (XII) 335-366 (2010). http://dx.doi.org/10.1366/000370210793561691

[4] D. W. Hahn, N. Omenetto, "Laser-induced Breakdown Spectroscopy (LIBS), Part II: Review of instrumental and methodological Approaches to Material Analysis and Applications to Different Fields", App Spectroscopy 66, (IV) 347-419 (2012). http://dx.doi.org/10.1366/11-06574

[5] V. Juvé, R. Portelli, M. Boueri, M. Baudelet, J. Yu, "Space-resolved analysis of trace elements in fresh vegetables using ultraviolet nanosecond laser-induced breakdown spectroscopy", Spectrochim. Acta Part B 63, 1047-1053 (2008). http://dx.doi.org/10.1016/j.sab.2008.08.009 
[6] W. Lei, V. Motto-Ros, M. Boueri, Q. ma, D. Zhang, L. Zheng, H. Zeng, J. Yu, “Time-resolved characterization of laser-inducced palsma from fresh potatoes". Spectrochim. Acta Part B 64, 891898 (2009)http://dx.doi.org/10.1016/i.sab.2009.07.015

[7] S. Beldjilali, D. Borivent, L. Mercadier, E. Mothe, G. Clair, J. Hermann, "Evaluation of minor element concentrations in potatoes using laser-induced breakdown spectroscopy", Spectrochim. Acta Part B 65, 727-733 (2010). http://dx.doi.org/10.1016/i.sab.2010.04.015

[8] J. Musilová, T. Tóth, J. Árvay "Contents of heavy metals in different saccharides fractions of potato tubers" Czech J. Food Sci 27, (Special Issue) 382-385 (2009).

[9] K. A. Anderson, B. A. Magnuson, M. L. Tschirgi, B. Smith, "Determining the Geographic Origin of Potatoes with Trace Metal Analysis Using Statistical and Neural Network Classifiers" J. Agric. Food Chem. 47, 1568-1575 (1999). http://dx.doi.org/10.1021/if980677u

[10] M. Garcimuño, D. M. Díaz Pace, G. Bertuccelli, "Laser-induced breakdown spectroscopy for quantitative analysis of copper in algae", Optics \& Laser Technology 47, 26-30 (2013).

[11] J. M. Lavornia, M. Garcimuño, M. J. Kristensen, G. Bertuccelli, "Relación entre la diversidad de comunidades Liquénicas saxícolas y la presencia de Contaminantes en Tandil, Buenos Aires. Resultados preliminares". XXV Reunión Argentina de Ecología (2012). Libro de Resúmenes. Universidad Nacional de Luján, Argentina

[12] D. M. Díaz Pace, C. A. D’Angelo, M. Garcimuño, G. Bertuccelli. “Análisis cuantitativo de Mg en agua natural mediante la técnica LIBS”, Opt. Pura Apl. 44, (IV) 719-729 (2011).

[13] http://physics.nist.gov/PhysRefData/ASD/lines form.html

\section{Introducción}

La creación del laser en la segunda mitad del siglo XX, ha dado origen a numerosas y variadas técnicas de medición. En particular, enfocando un pulso de radiación sobre la superficie de una muestra se genera un plasma, el cual puede ser posteriormente estudiado espectroscópicamente analizando su espectro de emisión o de absorción. Este método se denomina LIBS (Laser-Induced Breakdown Spectroscopy) y ha sido muy difundido en las últimas décadas en diversas aplicaciones [1].

La puesta a punto de la técnica para el análisis cuantitativo, es un problema complejo. Sin embargo, para muchas prestaciones como las requeridas en el área de biomonitoreo y fitorremediación, LIBS puede utilizarse como complementaria a otras técnicas analíticas convencionales.

Considerando el uso de un laser pulsado, cuando el pulso electromagnético incide sobre la muestra, se produce una ablación del orden de los microgramos de masa. La materia absorbe la radiación, calentándose hasta alcanzar un estado de plasma (alta densidad iónica y electrónica), comenzando simultáneamente a irradiar luz. El plasma evoluciona temporalmente, expandiéndose y enfriándose. Esta evolución temporal, puede ser registrada espectralmente evidenciando la presencia de una señal de fondo o continuo durante los primeros cientos de nanosegundos de vida del plasma. La discriminación temporal de la señal permite registrar las líneas espectrales una vez que el continuo ha disminuido, lo que es muy importante cuando es necesario detectar elementos en concentraciones traza.

En los espectros de emisión obtenidos usando LIBS, hay abundante información sobre la composición de la muestra, pero es necesario conocer con cierto detalle la dinámica y evolución del plasma para poder realizar un análisis cuantitativo preciso. Los espectros atómicos están dominados por un conjunto de líneas espectrales, cuyas longitudes de onda brindan información acerca de los elementos presentes en las muestras analizadas y la intensidad de línea se corresponde con la concentración del analito en el plasma, lo que puede correlacionarse con la concentración en la muestra.

Entre los parámetros que caracterizan un plasma están la temperatura T y la densidad electrónica $\mathrm{N}_{\mathrm{e}}$. En el caso de plasmas producidos por láser, $\mathrm{T} \sim 1 \mathrm{eV} \mathrm{y} \mathrm{N} \sim 10^{16} \mathrm{~cm}^{-3}$. A partir de las ecuaciones de Boltzmann y Saha-Boltzmann que relacionan las poblaciones de los distintos niveles de energía, pueden ser estimados $\mathrm{T}$ y $\mathrm{N}$. Además, el estudio de los perfiles de línea permite conocer el grado de homogeneidad y absorción del plasma, así como también, determinar la Ne a partir del ancho de línea [2-3]. 
Las ventajas de usar esta técnica espectroscópica residen fundamentalmente en la simplicidad del análisis, la mínima preparación de las muestras, la mínima cantidad de masa necesaria para generar el plasma, el bajo costo y la posibilidad de realizar mediciones in-situ.

Por su versatilidad, la técnica LIBS puede ser implementada en una extensa variedad de aplicaciones relacionadas con el estudio del medio ambiente, desde el análisis de algas y líquenes hasta el análisis de agua o sedimentos [4]. En particular, es de interés principalmente en la agricultura, monitorear la presencia de ciertos nutrientes (como por ejemplo el nitrógeno) para optimizar las aplicaciones de fertilizantes y herbicidas. El uso de este tipo de agroquímicos, puede cargar el sistema con elementos tóxicos en concentración suficiente como para contaminar el suelo, el agua y consecuentemente los cultivos. Por este motivo, el análisis de la composición química de los vegetales resulta de vital importancia tanto para evaluar la calidad de los alimentos, como para conocer la sanidad del ambiente. La posibilidad de realizar un seguimiento frecuente de la concentración de nutrientes en el suelo y el cultivo, puede favorecer a una mejora en la estimación de la dosis de fertilizantes necesaria sin llegar a extremos de carencia o sobreabundancia que pudiesen perjudicar el rendimiento de la cosecha.

Los vegetales, a través del sistema radical, pueden absorber los minerales presentes en el suelo. En el caso de las hortalizas que crecen en forma subterránea como es el caso de los tubérculos, el rabanito o la zanahoria, es posible que exista también, una difusión de los elementos desde el suelo hacia el interior del vegetal a través de la cáscara. Dado que los tubérculos son la reserva de nutrientes de la planta, es allí donde pueden bioacumularse elementos que no son necesarios o incluso resultan tóxicos para la planta o para su posterior consumo. Hay trabajos reportados en la literatura donde el análisis LIBS aplicado en papas ha permitido detectar hasta 27 elementos (mayoritarios y trazas), en la cáscara y en el interior del tubérculo [5]. Algunos trabajos reportan además los valores estimados de $\mathrm{T}$ y $\mathrm{N}_{\mathrm{e}}$ a partir de las líneas detectadas [6] e incluso se han desarrollado algoritmos para el análisis cuantitativo [7]. El análisis del almidón reviste una importancia adicional ya que puede ser utilizado como floculante en el proceso de saneamiento y potabilización de agua. En el trabajo de J. Musilová et. al [8] se presentan resultados de las determinaciones de tres metales pesados $(\mathrm{Cd}, \mathrm{Cu}, \mathrm{Zn})$ en diferentes fracciones de almidón, sacáridos solubles e insolubles en agua. Por otro lado, la determinación de los elementos que componen la papa pueden ser correlacionados con el lugar de procedencia del cultivo, lo que también ha sido testeado usando análisis LIBS [9]. Habiendo aplicado con anterioridad esta técnica espectroscópica al estudio de algas fluviales [10] y líquenes saxícolas [11], se decidió analizar papas a fin de disponer de una herramienta simple para la detección de metales pesados y/o vestigios de agroquímicos.

En este trabajo se presenta un estudio de la composición de los diferentes tejidos constitutivos de la papa usando LIBS. El principal objetivo de la investigación fue lograr implementar esta técnica y determinar protocolos para la preparación de muestras adecuadas a fin de realizar un análisis cualitativo. La preparación de las muestras consistió en la recolección, disecado, molienda y compactación del vegetal, fabricando pastillas sólidas y resistentes. Se consideró además, un análisis diferenciado en cada parte del vegetal: cáscara, pulpa, almidón y jugo.

\section{Arreglo experimental}

\section{2.a. Preparación de las muestras}

Antes de realizar el análisis LIBS, las papas fueron lavadas y peladas. Las distintas partes del tubérculo fueron separadas para la preparación de las distintas muestras. Usando una máquina extractora de jugo, se exprimió la papa, la pulpa fue colectada por separada y se dejó reposar el líquido en otro recipiente. Al cabo de un par de horas, el sistema coloidal se separó por sedimentación natural en dos fases: una líquida de color oscuro que flotaba sobre otra fase sólida color blanquecino que es el almidón. El jugo fue extraído usando una jeringa y mezclado con óxido de calcio (6 g de CaO Aldrich Powder 99,9 \%; 5,5 ml de líquido) a fin de obtener una matriz sólida en forma de pastilla de $3 \mathrm{~cm}$ de diámetro y $1 \mathrm{~cm}$ de espesor. Este procedimiento fue descripto en detalle anteriormente, en el trabajo publicado por Díaz Pace et al. [12] y está esquematizado de manera simplificada en la Fig. 1.

Se preparó una solución usando agua destilada y parte del almidón extraído de la papa. Se colocó esta mezcla en una caja de Petri, esparciéndola en forma de capa delgada sobre el fondo del recipiente contenedor. Luego fue calentado en microondas por $20 \mathrm{~s}$ al $100 \%$ de potencia formando un film delgado, 
el cual se dejó enfriar a temperatura ambiente. Se lograron fabricar tres discos de este tipo, con características muy similares.

Por otro lado, la pulpa y la cáscara, fueron compactadas separadamente en forma de pastillas usando el mismo molde antes mencionado.

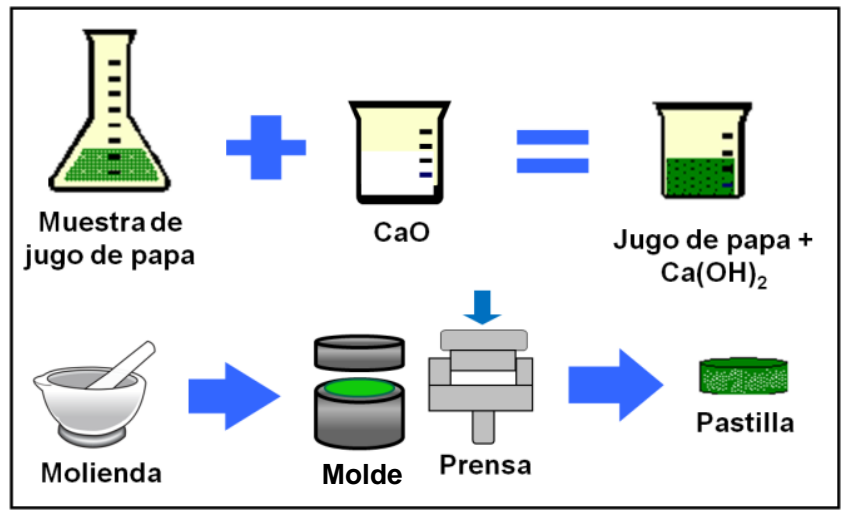

Fig 1. - Esquema de preparación de las muestras
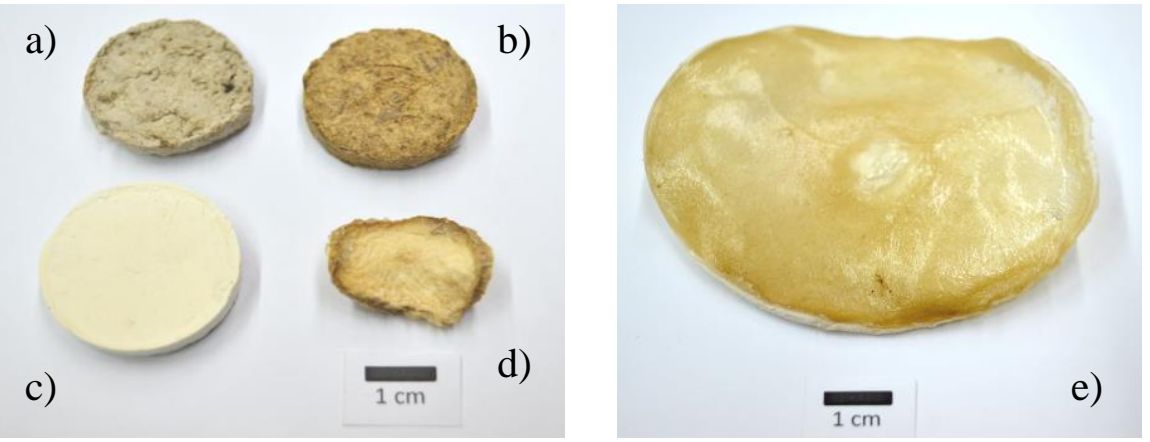

Fig. 2 - Muestras de papa obtenidas: a) pulpa, b) cáscara, c) jugo mezclado con CaO, d) rodaja de papa y e) almidón

\section{2.b. Equipo experimental}

Para la realización de este trabajo fueron empleadas dos configuraciones experimentales con distintas prestaciones. En primer lugar se realizó un examen preliminar de las muestras, usando un equipo sin resolución temporal con el cual pudo ser registrado el espectro de emisión en el rango 250-775 nm. Posteriormente, para analizar elementos traza y detectar la posible presencia de metales pesados, se utilizó un equipo con mayor resolución y discriminación temporal.

\section{2.b.1- Configuración sin discriminación temporal}

Los plasmas fueron generados en aire a presión atmosférica enfocando la radiación de un láser pulsado Nd:YAG (Continuum Surelite II, $\lambda=1064 \mathrm{~nm}, 7 \mathrm{~ns}, 100 \mathrm{~mJ} /$ pulso, frecuencia de disparo $2 \mathrm{~Hz}$ ) en la superficie de las muestras mediante una lente de cuarzo de $10 \mathrm{~cm}$ de distancia focal. Se decidió trabajar con un plasma alargado (ver Fig. 3), lo que se logró al desenfocar el spot del láser aumentando la distancia entre la muestra y la lente. En esta configuración el plasma es más estable, pero puede haber una contribución del aire circundante, lo que debe ser tenido en cuenta en el análisis espectral. 


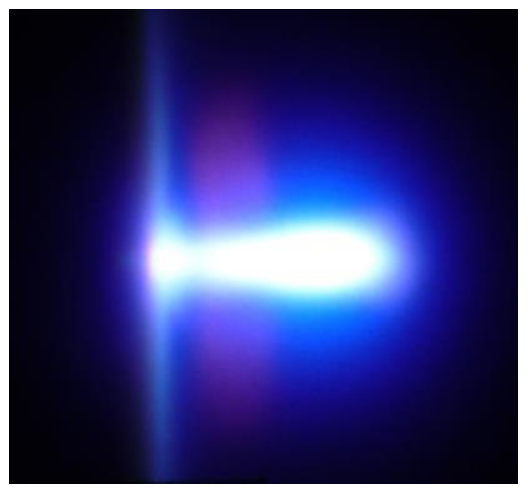

Fig. 3 Plasma producido en aire formado sobre la superficie de la muestra.

Las distintas pastillas fueron fijadas a un soporte rotatorio, que giraba continuamente para evitar que sucesivos disparos del laser provocasen la formación de un cráter. Cuando esto último sucede, el plasma queda parcialmente confinado en el interior del cráter lo que redunda en el apantallamiento de la señal observada sobre la rendija del monocromador.

La radiación emitida por el plasma fue observada perpendicularmente a la dirección del haz laser enfocándola con una lente de cuarzo de $20 \mathrm{~cm}$ de distancia focal en la rendija de entrada de un espectrómetro (Acton Research Corporation configuración Czerny Turner, de $390 \mathrm{~mm}$ de distancia focal, red holográfica de 1200 líneas $/ \mathrm{mm}$ ). El detector utilizado fue un arreglo de 1024 diodos OMA, modelo RY1024; 24,5 mm de longitud. Los datos fueron adquiridos mediante una interface conectada a la PC (modelo ST- 120, Princeton-Instruments Inc) y posteriormente analizados.

\section{2.b.2- Configuración con discriminación temporal}

Con el propósito de detectar elementos traza, se ensayó otra configuración experimental reemplazando el espectrómetro por un monocromador (Jovin Yvon modelo THR, configuración Czerny-Turner, distancia focal 1,5 m, red de difracción holográfica de 2400 líneas/mm, resolución 300000). Se eligió como detector un fotomultiplicador (Hamamatsu IP28) y la señal fue procesada mediante un Box-Car (Stanford Research Systems) que permite seleccionar la ventana temporal en la cual la señal es integrada y promediada en forma sincronizada con un pulso eléctrico proveniente del laser (trigger). Finalmente, la señal fue procesada usando una PC.

a)

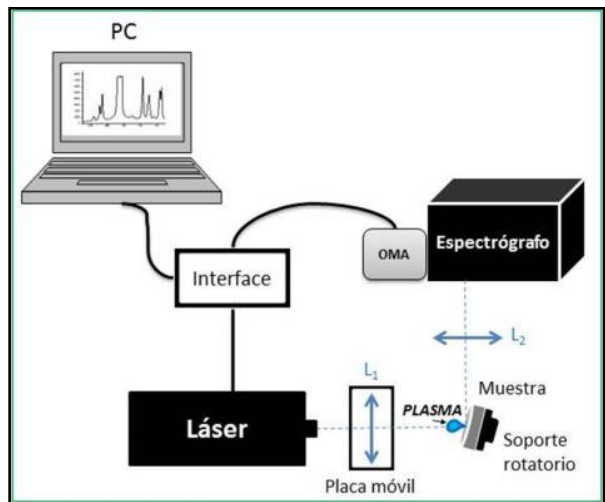

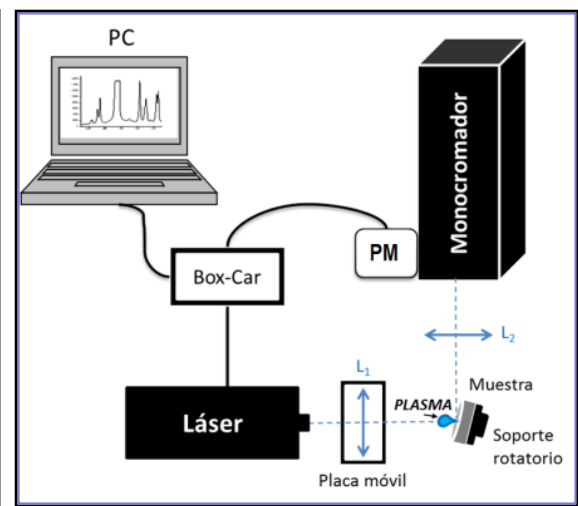

b)

Fig. 4 Configuraciones experimentales utilizadas a) sin resolución temporal; b) con resolución temporal

\section{Resultados}

Los distintos procedimientos utilizados para la preparación de las muestras, permitieron fabricar pastillas con superficies de distinta calidad y rugosidad. Mientras que la rodaja de papa y el almidón se flexionan en el proceso de secado, las muestras de cáscara y pulpa ofrecen superficies desunidas que son muy endebles frente a la acción del laser. Este tipo de pastillas no son tan convenientes de ser utilizadas en experiencias LIBS dado que introducen un factor de variabilidad adicional reduciendo consecuentemente la sensibilidad y precisión analítica de la técnica. Por otro lado, la pastilla fabricada mezclando el jugo con el 
oxido de calcio, es más dura y resistente a los disparos del laser y ofrece una superficie lisa sobre la cual producir el plasma. Tras una gran cantidad de disparos, la superficie puede ser alisada nuevamente usando una lija fina permitiendo así, ser utilizada por más tiempo que las demás muestras. Una ventaja de este tipo de muestras, es que facilita las determinaciones de concentración de los elementos porque permite utilizar pastillas de calibración fabricadas a partir de soluciones de los elementos de interés y el mismo CaO. Para realizar este tipo de comparaciones entre muestras de diferentes naturalezas, es imprescindible demostrar antes la equivalencia de las matrices, lo cual fue corroborado para el caso de algas en el trabajo antes mencionado. [10]

\section{3.a Resultados obtenidos con el espectrómetro, sin resolución temporal}

Los elementos que pudieron ser identificados en todos los tejidos son $\mathrm{Mg}, \mathrm{Si}, \mathrm{Ca}, \mathrm{Ni}, \mathrm{Al}, \mathrm{Cu}, \mathrm{Cr}, \mathrm{Sr}, \mathrm{Ba}, \mathrm{Na}, \mathrm{N}$, $\mathrm{H}$, Li. Las longitudes de onda medidas de los elementos detectados fueron comparadas con las de la base de datos electrónica del NIST [13] y aparecen listadas en la Tabla 1.

TABLA 1. Elementos detectados y líneas espectrales seleccionadas para el análisis LIBS

\begin{tabular}{|c|c|}
\hline $\begin{array}{l}\text { Elemento } \\
\text { detectado }\end{array}$ & $\begin{array}{l}\text { Longitud de onda } \\
\text { (nm) }\end{array}$ \\
\hline \multirow[t]{2}{*}{ Mg II } & 279.55 \\
\hline & 280.27 \\
\hline $\mathrm{Mg} \mathrm{I}$ & 285.21 \\
\hline Si I & 288.16 \\
\hline \multirow{4}{*}{ Ca II } & 315.89 \\
\hline & 317.93 \\
\hline & 393.37 \\
\hline & 396.85 \\
\hline \multirow{3}{*}{$\mathrm{Ni} \mathrm{I}$} & 323.29 \\
\hline & 336.96 \\
\hline & 339.11 \\
\hline \multirow{2}{*}{$\mathrm{Al} \mathrm{I}$} & 308.22 \\
\hline & 394.40 \\
\hline \multirow{2}{*}{$\mathrm{Cu} \mathrm{I}$} & 324.75 \\
\hline & 327.40 \\
\hline \multirow{3}{*}{$\mathrm{Cr}$} & 357.8 \\
\hline & 425.44 \\
\hline & 427.48 \\
\hline \multirow{2}{*}{ Sr II } & 407.77 \\
\hline & 421.55 \\
\hline \multirow{2}{*}{ Fe I } & 430.79 \\
\hline & 432.58 \\
\hline \multirow{2}{*}{ Ba II } & 455.40 \\
\hline & 493.41 \\
\hline \multirow{2}{*}{$\mathrm{Na} I$} & 588.99 \\
\hline & 589.59 \\
\hline \multirow{4}{*}{$\mathrm{N}$} & 575.25 \\
\hline & 742.36 \\
\hline & 744.22 \\
\hline & 746.83 \\
\hline $\mathrm{H}_{\alpha}$ & 656.27 \\
\hline \multirow{2}{*}{$\mathrm{Li}$} & 670.78 \\
\hline & 670.79 \\
\hline \multirow{3}{*}{0} & 777.20 \\
\hline & 777.42 \\
\hline & 777.54 \\
\hline
\end{tabular}


En la Fig. 5 se puede observar el espectro completo en el rango 250-775 nm, el cual fue registrado en sucesivas mediciones en intervalos de $50 \mathrm{~nm}$, usando el espectrómetro y el analizador multicanal. Cada registro es el resultado de la acumulación de 10 pulsos, lo que permite minimizar las fluctuaciones.
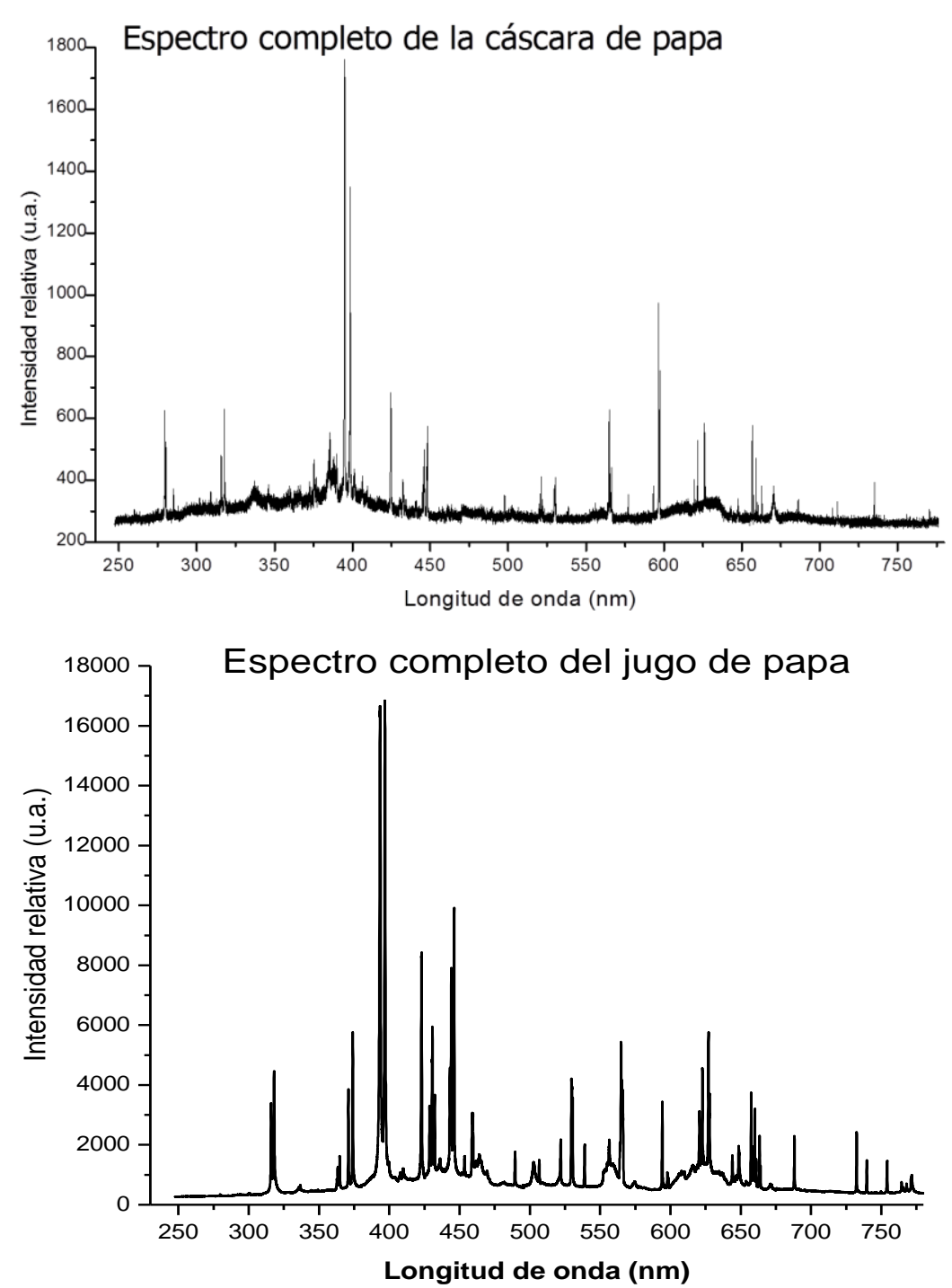

Fig. 5 Espectros de emisión del jugo y de la cáscara de papa en el rango 250-775 nm.

Como puede observarse en los gráficos de la Fig. 6, existen diferencias en los espectros de las distintas partes de la papa, lo que indica la variación de composición de los tejidos. El espectro obtenido en la muestra de pulpa es muy similar al obtenido en la muestra de almidón, en la mayor parte del espectro. Esto puede atribuirse a que la extracción mecánica del jugo no logra separar todo el almidón contenido en la pulpa, sino principalmente el que se haya disuelto en agua (que posteriormente precipita por decantación natural). 

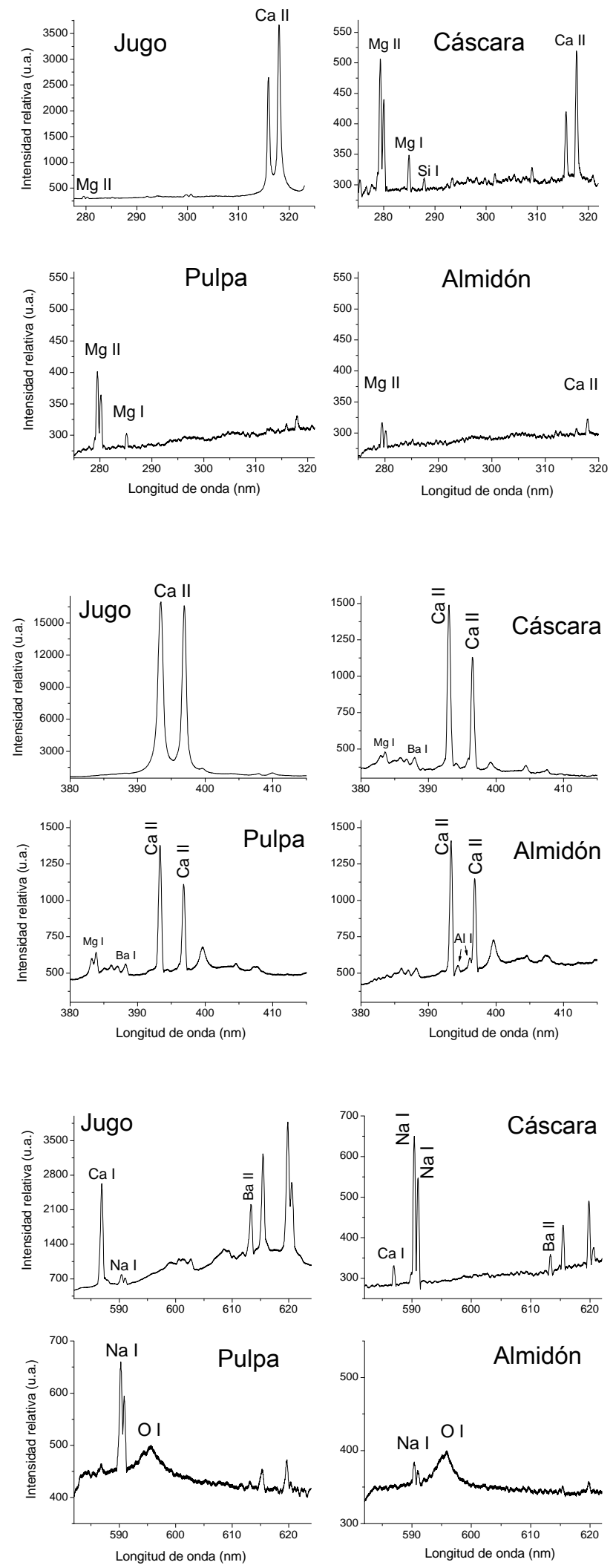

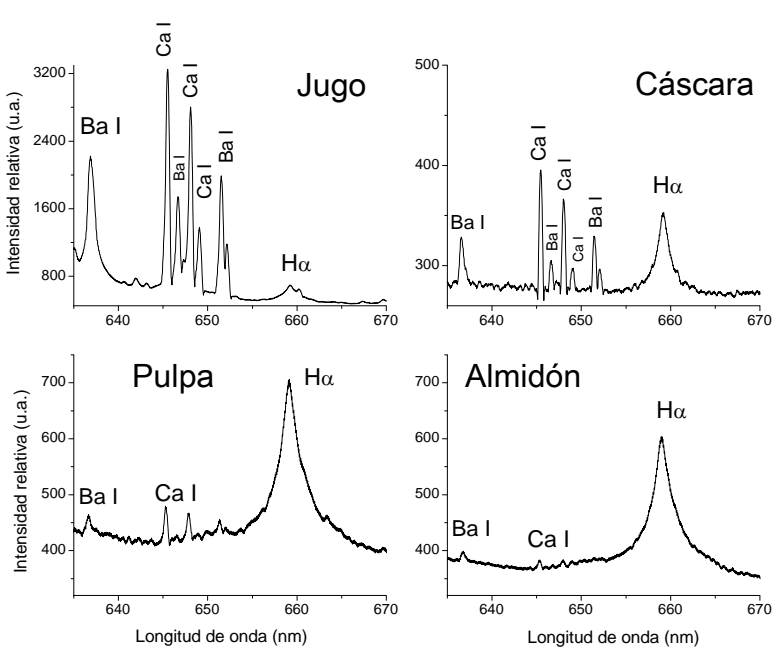

Fig. 6 Espectro de emisión de los distintos tejidos constitutivos de la papa en algunos rangos del espectro.

En el rango de 275-325 nm se observa un par de líneas resonantes de Mg II y otra de Mg I, presentes en los cuatro tipos de muestras. La intensidad relativa indica que la concentración de este elemento es mayor en la cáscara que en los demás tejidos. También es posible identificar en la muestra de cáscara una de las líneas más intensas de silicio.

Las líneas resonantes de Ca II dominan en intensidad los espectros obtenidos en la muestra de jugo, dado que esta sustancia fue mezclada con el CaO. Es importante considerar, que el continuo de las líneas de de Ca II, pueden crear interferencias con líneas de otros elementos de interés que sean muy próximas en longitud de onda.

\section{3.b Medición con el monocromador}

Se registraron perfiles de líneas de los distintos elementos. Los que pudieron ser detectados con una buena relación señal ruido son $\mathrm{Sr}, \mathrm{Ba}, \mathrm{Cu}, \mathrm{Si}, \mathrm{Cr}, \mathrm{Ni}, \mathrm{Al}$. En cambio la intensidad de las líneas de Ti, Fe, Mn, $\mathrm{K}, \mathrm{V}, \mathrm{Mo}$, es muy débil respecto del ruido, lo que indica que la concentración de estos elementos es menor.

También se registraron los espectros temporales de las líneas de $\mathrm{Sr}, \mathrm{Mn}, \mathrm{Ni}, \mathrm{Cu}, \mathrm{Al}$, Si. Del análisis temporal puede decirse que $\mathrm{Mn}, \mathrm{Ni}, \mathrm{Cu}$ tienen una mayor intensidad en los primeros tiempos (1-4 $\mu \mathrm{s})$. En cambio las líneas de $\mathrm{Al} \mathrm{y} \mathrm{Si,} \mathrm{aparecen} \mathrm{después} \mathrm{de} \mathrm{los} 4 \mu \mathrm{s}$.

Se realizaron barridos con el monocromador en las zonas de los espectros registrados con el espectrómetro donde había dudas respecto a los elementos presentes. Sin embargo, muchas de las líneas observadas todavía no pudieron ser correctamente identificadas, por lo que sería necesario contar con muestras de referencia para comparar la información espectral.

El almidón es la muestra que menor cantidad de elementos contiene y la cáscara la de mayor diversidad de ellos, particularmente $\mathrm{Mg}$, Na y Ca.

A partir de las líneas de los elementos detectados, podría realizarse la caracterización del plasma. Conociendo los parámetros espectroscópicos, que incluyen la longitud de onda, la probabilidad de transición, el valor de los niveles de energía y el factor de degeneración de los mismos, es posible estimar la temperatura del plasma realizando para ello un plot de Boltzmann. La densidad electrónica podría ser determinada a partir del ancho de línea.

\section{Conclusiones}

La técnica LIBS pudo ser implementada eficientemente al análisis de papa, lo que constituye un aporte al estudio de vegetales usando plasmas producidos por laser. Se ensayaron con éxito distintos tipos de muestra de acuerdo a la parte del tubérculo que se pretendía estudiar y los componentes mayoritarios y minoritarios pudieron ser identificados.

Poder obtener a partir del jugo de papa las muestras sólidas fabricadas con CaO es una gran ventaja cuando no se disponen de muestras de referencia certificadas, como sucede muchas veces en el análisis de 
vegetales. Es necesario continuar estudiando las condiciones óptimas para la ablación y detección de la radiación, a fin de mejorar la performance analítica de LIBS que está limitada por los efectos de matriz y las características de la compactación y superficie de la muestra.

Como continuidad del trabajo, pueden establecerse relaciones semicuantitativas respecto de un elemento de referencia, a fin de hallar nuevos indicadores en el estudio de la sanidad del suelo y de los cultivos.

\section{Agradecimientos}

Este trabajo fue realizado en el Instituto de Física 'Arroyo Seco' (IFAS) de la Facultad de Ciencias Exactas de la UNCPBA, mediante una beca de postgrado otorgada por el Consejo Nacional de Investigaciones Científicas y Técnicas (CONICET). 